\title{
Fibre Laser Doppler Vibrometry System for Target Recognition
}

\author{
Michael P. Mathers ${ }^{\mathrm{a}}$, Samuel Mickan ${ }^{\mathrm{a}}$, Werner Fabian ${ }^{\mathrm{c}}$ Tim McKay $^{\mathrm{b}}$ \\ ${ }^{\text {a }}$ School of Electrical and Electronic Engineering, The University of Adelaide, North Terrace, \\ Adelaide, SA, Australia 5005; \\ ${ }^{\mathrm{b}}$ Electronic Warfare and Radar Division, Defence Science and Technology Organisation, \\ PO Box 1500, Edinburgh, SA, Australia 5111; \\ ${ }^{\mathrm{c}}$ Laser Integrated Technologies, 20 Arafura Court, Hallett Cove, SA, Australia, 5158
}

\begin{abstract}
Vibrations from a target provide a difficult to mask target signature. Vibrometry shows potential for long-range target identification whilst a fibre implementation may lead to a smaller, more compact system when compared with an equivalent solid-state source solution. A prototype fibre LDV system and electronic demodulation scheme using lowcost telecommunications components is described and tested. The aim of the system is to remove the velocity component of a target signature for target identification purposes. Signal processing methods and signature measurements are described which demonstrate the utility of the system for target recognition.
\end{abstract}

Keywords: Doppler, Fibre-optics, Signal Processing, Vibrometry

\section{INTRODUCTION}

Laser Doppler Vibrometry (LDV) offers the potential to simultaneously classify a target whilst measuring its range and velocity. LDV is a technique for identifying a target based on its vibration spectrum. LDV measures the Doppler shifts, relative to a transmit/receive configuration. Target identification of moving vehicles is made more difficult due to frequency shifting caused by the target velocity. Simpler identification algorithms can be used if the vibration spectra of a stationary and moving target can be measured independent of velocity.

This paper reports the development of a prototype identification system based around the Laser Doppler Vibrometry (LDV) technique. The prototype was developed to demonstrate Doppler vibration measurements, the identification of moving targets and to test range measurement methods. Note that range measurement is not discussed in this paper.

A common architecture is to use a heterodyne receiver, perhaps in conjunction with a narrow locking phase-locked loop (PLL), to demodulate the return signal and serve as the input to a data acquisition device. For this system, a PLL, capable of locking over a large range (20-50 MHz), is used to perform the demodulation in order to allow the detection of targets with a range of velocities. 


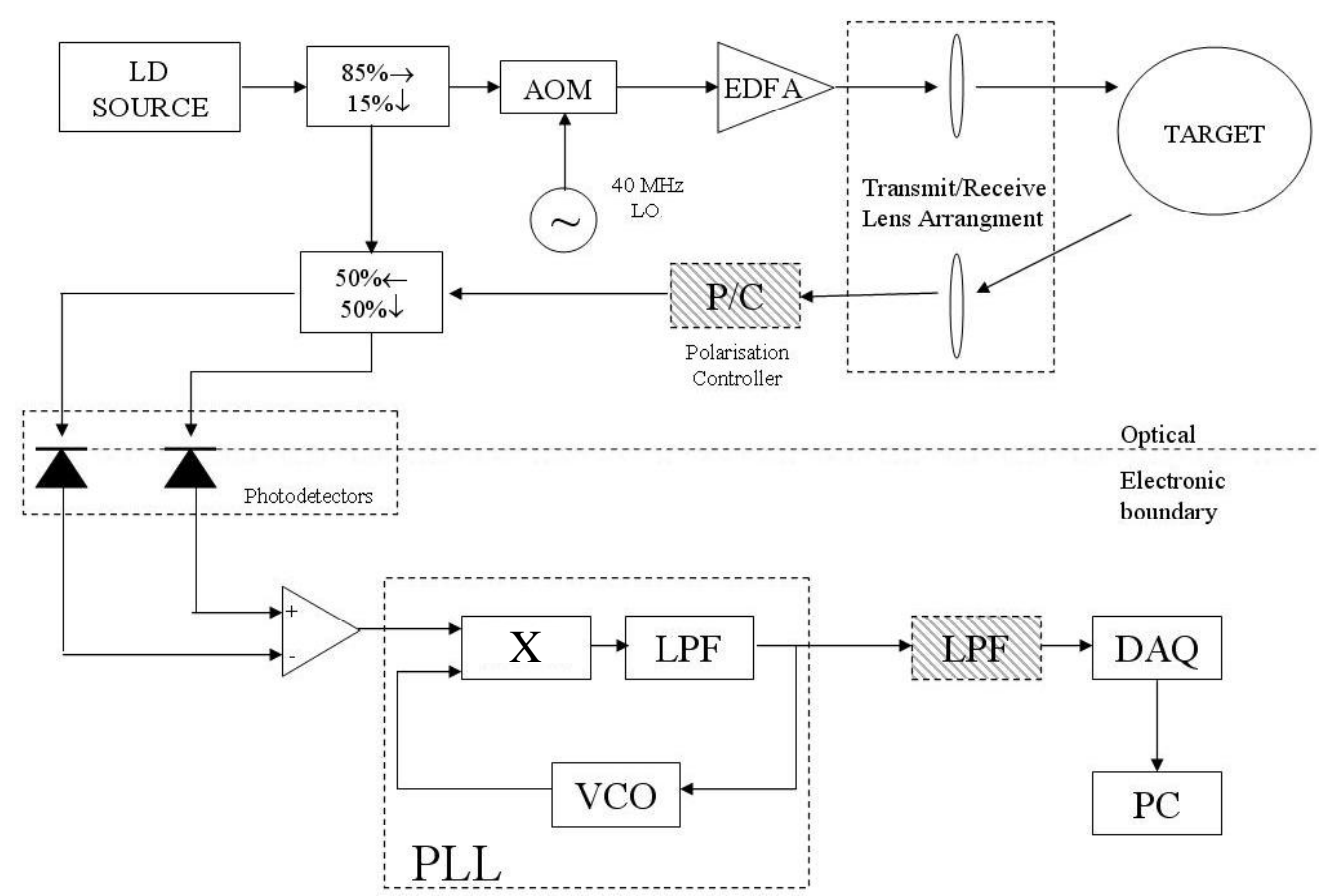

Figure 1: Block diagram of the prototype system. The blocks with percentages and arrows represent a variable ratio coupler $(85 \%-15 \%)$ and a fixed 50-50 coupler $(50 \%-50 \%)$. The grey-hashed boxes indicate components that were not always used as part of the system.

The important feature of this system is the design of the receiver electronics. The vibration information is recovered from the output of the PLL low pass filter (LPF). The advantage of this approach is that signatures from targets travelling with velocities anywhere between -45 and $+45 \mathrm{~km} / \mathrm{hr}$ can be mixed out. Expanding the locking range of the PLL can increase the range of velocities that can be mixed out. The disadvantage and challenge is that accommodation of a large range of target speeds requires a large detection bandwidth thus increasing the receiver noise floor and dramatically lowering the effective sensitivity of the receiver.

Identification of objects by looking at acoustic or vibration information is not a new field but has been investigated for some time now.

Olsson shows that information received from interrogating the target at different angles is similar enough to be perceived as the identifying information. Further to this he provides evidence that vehicles have a unique signature that may be used for identification purposes [1].

Letalick, et al demonstrated a fibre-laser-based system with an output power of 2 Watts. The results obtained were for ranges up to 100 metres with little signal processing at the receiver end [2].

The output powers achieved using fibre lasers are still increasing with narrow spectral width sources now reaching 80 Watt output powers [3].

Commercial systems for vibrometry measurements exist. Polytec produces vibrometry systems capable of measuring vibrations at the picometre scale.[4]. It is important for the system to differentiate between the Doppler vibration spectrum, used for target discrimination, and the net Doppler shift which describes the target velocity. Commercial systems such as, Polytec's VDD digital laser vibrometer, typically only work for a very small range of target velocities (net Dopper shift corresponding to less than 3 kilometres per hour) and over a very small range (less than 5 metres). 


\section{THEORY}

\subsection{Laser Doppler Vibrometry}

Laser Doppler Vibrometry (LDV) is a technique that uses the Doppler frequency shifts of a laser carrier, present after reflection from a moving surface, to detect and measure the vibrations present on a surface.

It can be shown that the receiver perceives a frequency shift, $\Delta f$, with respect to the source, if the target is moving at a speed $v$ towards the receiver. Assuming that the source and observer are at the same point [5]:

$$
\Delta f=\frac{2 V f_{0}}{c} \cos \theta=2 \frac{V}{\lambda_{0}} \cos \theta
$$

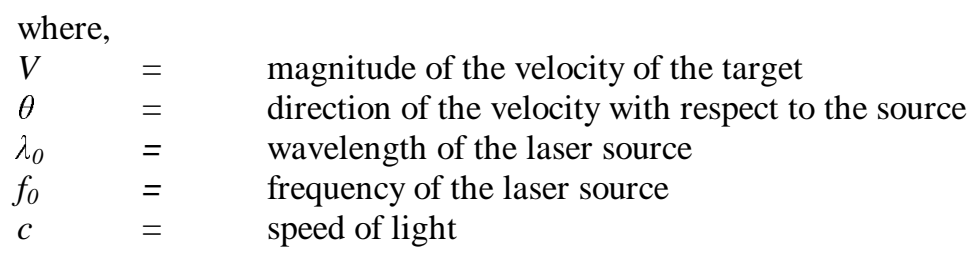

\subsection{Modelling a Vibrating Surface}

Consider a surface vibrating at a frequency $f_{S U R F A C E}$, the position of the surface relative to its rest position, $x(t)$, can be modelled using a sinusoid.

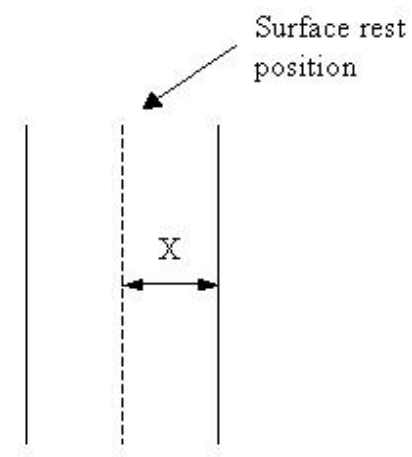

Figure 2: Model used for a vibrating surface.

$$
x(t)=X \sin \left(2 \pi f_{\text {SURFACE }} t\right)
$$

Since the surface has a velocity (displacement is time-varying), when the incident beam from the vibrometry system reflects from the surface a frequency shift will be imposed on the return signal. The magnitude of the frequency shift can be found using the Doppler shift equation (1).

$$
\Delta f=\frac{4 \pi X f_{\text {SURFACE }} \cos \left(2 \pi f_{\text {SURFACE }} t\right) \cos \theta}{\lambda_{0}}
$$

This equation predicts that the Doppler shift will be time varying with a frequency equal to the frequency of vibration $f_{\text {SURFACE. }}$. The magnitude of the shift will be proportional to the maximum deviation and the surface vibration frequency. 


\subsection{Signature Comparison}

A feature of the acquisition software is the ability to make a comparison between two recorded signatures and to infer whether the signatures come from the same target or from different targets.

The current comparison algorithm works by analysing the Fast Fourier Transform (FFT) of both samples and forming an estimate of the power spectral density for each sample. Before either FFT is calculated, the two signals are normalised to unit power. The absolute differences between the power spectral densities at each discrete point are calculated. Following this step, the mean and median values of the absolute differences are calculated. If both the mean and median values are less than a predetermined threshold (separate thresholds for mean and median) then the signatures are considered to have come from the same source. Thresholds are used because the amount of noise at each frequency will vary slightly for each sample taken. The thresholds were optimised experimentally to minimize incorrect classification for the signatures tested.

\section{EXPERIMENTS}

\subsection{Minimum and Maximum Detected Vibrational Frequencies}

Spectral components outside the limits of vibrational frequency detection are treated as spurious noise not contributing to the process of identifying the target. For this reason it is useful to know the system bandwidth in order to optimise algorithm efficiency. A signal generator was used to drive a speaker with a sinusoidal signal at low frequency. The frequency was lowered until the response was no longer clearly visible at the output of the PLL.

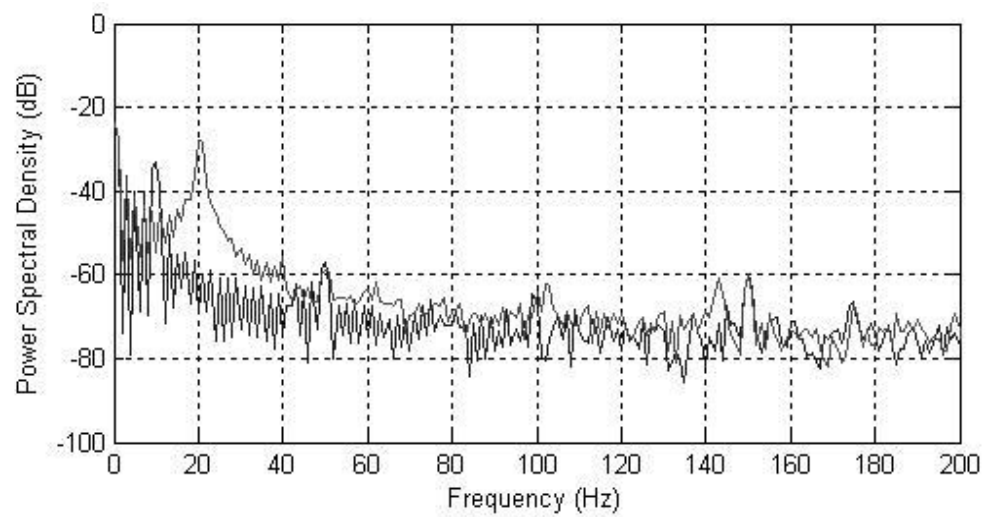

Figure 3: Two plots showing the FFT resulting from targeting a speaker vibrating at $10 \mathrm{~Hz}$ and $20 \mathrm{~Hz}$ for an observation duration of 1 second. A further filtering stage at the output of the PLL with a cut-off frequency of $3 \mathrm{kHz}$ was used to obtain these results.

The results show that frequencies below 10 to $20 \mathrm{~Hz}$ are difficult to detect without sampling for extended periods of time. As a result components below $10 \mathrm{~Hz}$ should be ignored when making signature comparisons, as the system cannot reliably detect components at those frequencies.

Raising the frequency showed a visible response from the speaker up to around $2 \mathrm{kHz}$. Beyond $2 \mathrm{kHz}$ the system response falls away. This suggests that components above $2 \mathrm{kHz}$ should not be considered as part of the vibration signature and should be ignored when making comparisons. This is not a limitation as a result from Wu reported 80 percent of vibration information power from a vehicle lies between 0 and $2 \mathrm{kHz}$ with 90 percent of power lying between 0 and $4 \mathrm{kHz}$ [6]. 


\section{VIBROMETRY RESULTS}

\subsection{Moving Target Vibrometry}

The purpose behind placing the PLL in the receiver was to be able to cope with moving targets and still receive a similar vibration signature to their stationary counterparts. To test this functionality a speaker was mounted above a stand with wheels for moving the speaker back and forth. The range to target from the transmit/receive structure was between 70 to 100 centimetres. To improve receiver signal-to-noise performance the speaker was fitted with retro tape to increase return power.
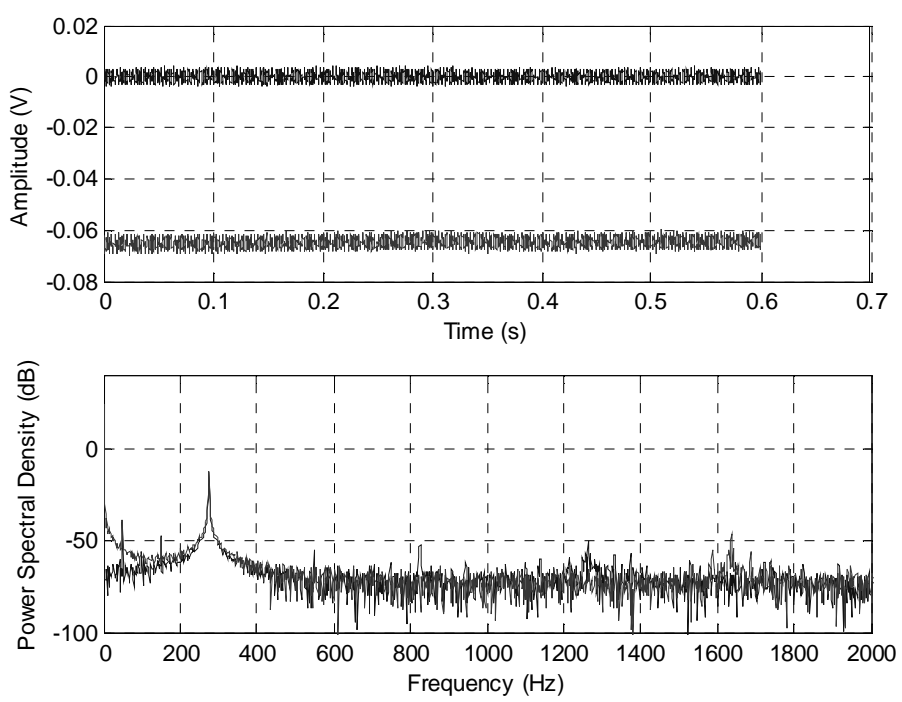

Figure 4: PLL output waveform and frequency responses for stationary (higher plot in time domain, lower in frequency domain for very low frequencies) and constant velocity targets. The target was a speaker, mounted on wheels, vibrating at $275 \mathrm{~Hz}$. Note the DC shift proportional to the velocity in the time domain and no shifting of the $275 \mathrm{~Hz}$ peak in the frequency domain. The 0.6 seconds of data has been extracted from a larger 2-second sample for illustration purposes. 

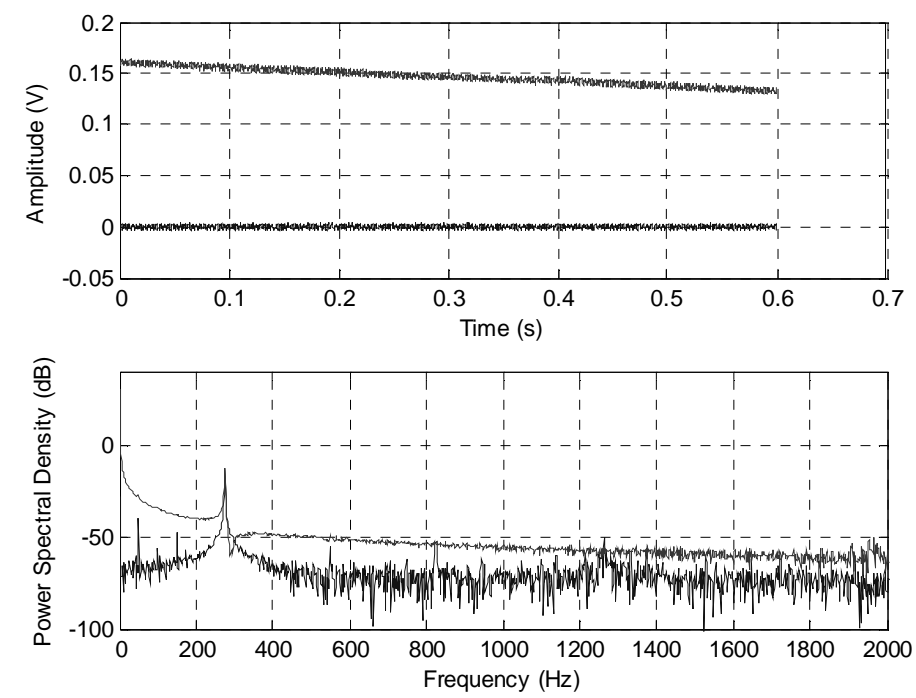

Figure 5: PLL output waveform and frequency responses for stationary (lower lines in both plots) and decelerating targets (top plots). The target was a speaker, mounted on wheels, vibrating at $275 \mathrm{~Hz}$. Note the DC shift proportional to the velocity in the time domain and no shifting of the $275 \mathrm{~Hz}$ peak in the frequency domain. The 0.6 seconds of data has been extracted from a larger 2-second sample for illustration purposes.

The results highlight some points to consider when comparing signatures using spectral information. The first is that the system will preserve the vibration information without any frequency shifts in the case of a moving target. However, the second point is that a moving target will have substantially different very low frequency (below $10 \mathrm{~Hz}$ ) components to a stationary signal due to the velocity producing a DC component. The results given above were not easily obtained either but required some post processing to remove samples were the PLL had lost lock or was trying to acquire lock. Figure 12 below shows the spectra using all the data from which a subset was chosen to produce Figure 10.

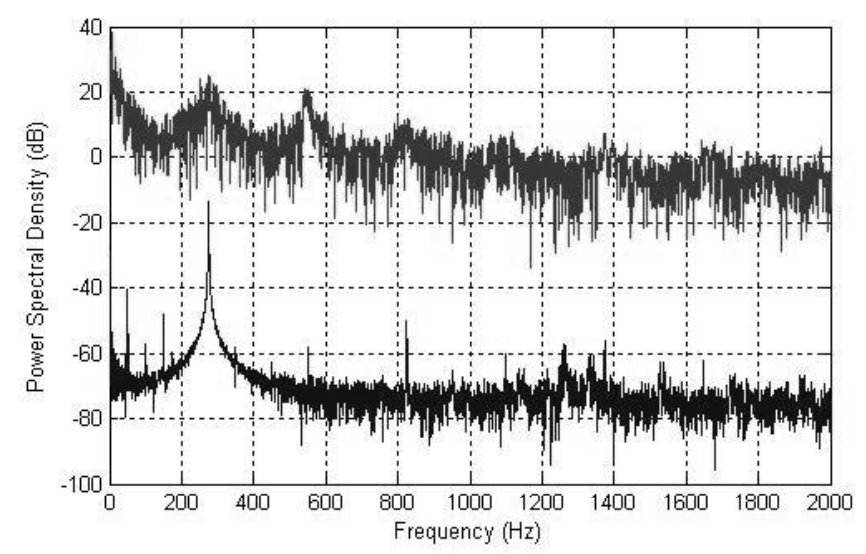

Figure 6: Frequency responses for the all the data collected in the same experiment as Figure 5. The extra noise in the top plot (moving target) can be attributed to a combination of points where the PLL has lost lock and points where unsteadiness in controlling the motion of the stand has added extra vibration and hence extra frequency components. 
It is clearly seen that losing lock is detrimental to correct operation of the system and would void any signature comparison. In future, the state of the PLL will be monitored, and data collected while out of lock will be discarded.

\subsection{Comparison of Signatures}

Since it was not possible to transport the prototype system outside for testing, vehicle sound recordings were used as an alternative method of testing the comparison algorithms. Recordings of vehicles revving engines or passing by were played through the speaker used for other experiments. For each recording, 3 half-second samples were taken and stored.

Once all the data had been collected, each sample was compared to each of the other samples, including itself, and the results of the comparisons were recorded in a spreadsheet table.

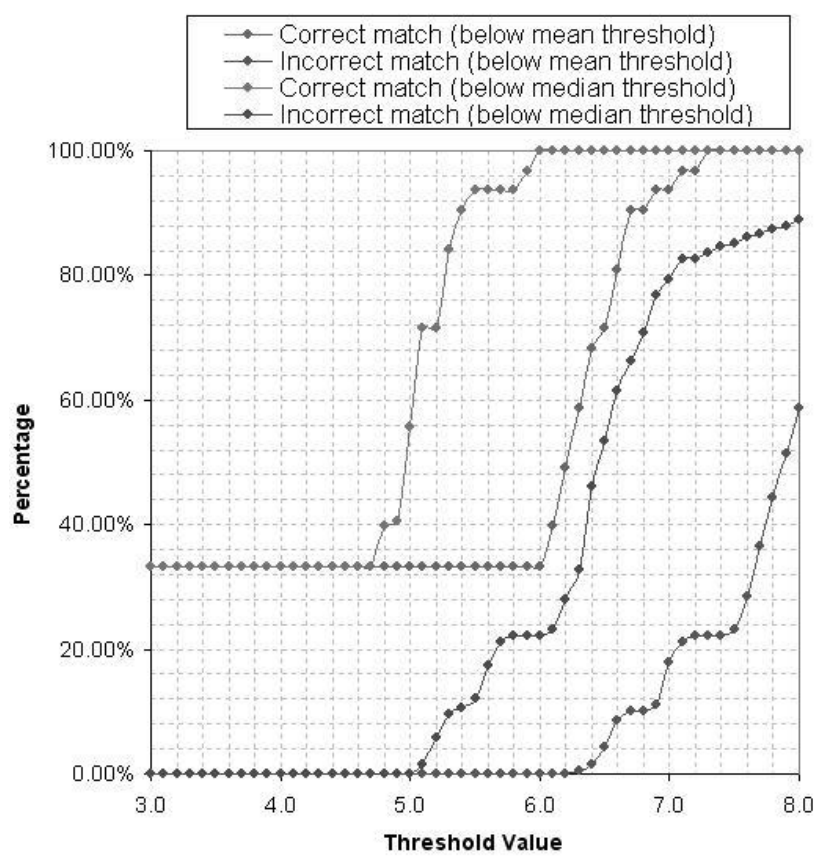

Figure 7: Plot showing the results from using different mean and median thresholds when comparing the sample signatures. Correct match means that the result when comparing two samples from the same source was below the threshold used. An incorrect match means that the result when comparing two samples from different sources was below the threshold.

Based upon the 21 samples collected, setting the mean absolute difference threshold to 6.8 and the median absolute difference threshold to 5.4 yielded a correct match with a stored sample in $90 \%$ of cases and positively matched with a stored sample incorrectly $10 \%$ of the time.

These numbers are a positive beginning for comparison of signatures based on a simplistic approach. More sophisticated approaches including excluding redundant parts of the frequency spectrum or other techniques entirely could improve on these results.

\section{CONCLUSIONS}

This project has demonstrated a prototype LDV system that uses a phase-locked loop (PLL), to demodulate target vibration signatures. 
The PLL has been used to return a vibration signature for a moving target that overlaps with a signature of the same stationary target. The overlap makes target identification algorithms based on frequency content much simpler and more accurate. The challenge involved with using a PLL in this manner was that a large input bandwidth was used and resulted in the addition of large amounts of noise compared to other systems of this type.

Finally, it has been shown that an FFT analysis technique offers a high degree of discrimination between the target signatures used.

\subsection{Future Work}

The system as it stands is not state-of-the-art. There are many areas, which can be improved.

The current prototype is limited in operation to short ranges due to low power. Development and integration of a high power, low noise, and narrow spectral width source should allow the system to interrogate target vehicles kilometres away.

Finally, advanced signal processing techniques should be applied to the receiver electronics and computer algorithms to reduce the performance requirements of the optical components to as low a level as possible. This will help keep the cost of the system to a minimum, as electronic components are much cheaper than their optical counterparts. Much more complex algorithms exist for signal classification and could be applied to increase the accuracy of the signature comparison process.

\section{ACKNOWLEDGEMENTS}

Michael Mathers would like to thank both the Defence Science and Technology Organisation and the University of Adelaide for organising and providing financial support for this research project.

\section{REFERENCES}

1. Olsson, A., Target Recognition by Vibrometry with Coherent Laser Radar. 2003, Linkopings. p. 60.

2. Letalick, D., et al. Target Recognition by Vibration Frequency Analysis - A Comparison of Laser Vibrometry and Acoustic Methods. in SPIE: Swedish Defence Research Agency (FOI).

3. Alegria, C., et al., 83-W Single-Frequency Narrow-Linewidth MOPA Using Large-Core Erbium-Ytterbium CoDoped Fiber. IEEE Photonics Technology Letters, 2004. 16(8).

4. Measurement Solutions Made Possible By Laser Vibrometry. 2003, Polytec.

5. Electronic Warfare and Radar Systems Engineering Handbook. 2002, Naval Air Warfare Center Weapons Division.

6. Wu, H., M. Siegel, and P. Khosla. Vehicle Sound Signature Recognition by Frequency Vector Principal Component Analysis. in IEEE Instrumentation and Measurement. 1998. St. Paul, Minnesota, USA: Carnegie Mellon University. 